\title{
VERITAS OBSERVATIONS OF THE UNUSUAL EXTRAGALACTIC TRANSIENT SWIFT J164449.3+573451
}

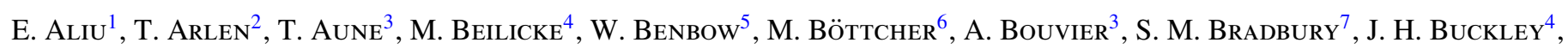
V. Bugaev ${ }^{4}$, A. Cannon $^{8}$, A. Cesarini ${ }^{9}$, L. Ciupik ${ }^{10}$, E. Collins-Hughes ${ }^{8}$, M. P. Connolly ${ }^{9}$, W. Cui ${ }^{11}$, R. Dickherber ${ }^{4}$, M. Errando ${ }^{1}$, A. Falcone ${ }^{12}$, J. P. Finle ${ }^{11}$, L. Fortson ${ }^{13}$, A. Furniss ${ }^{3}$, N. Galante ${ }^{5}$, D. Gall ${ }^{14}$, G. H. Gillanders ${ }^{9}$,

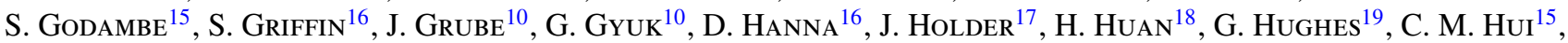

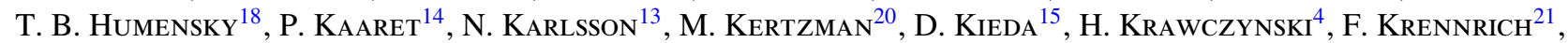
A. S. Madhavan ${ }^{21}$, G. Maier ${ }^{19}$, P. Majumdar ${ }^{2}$, S. McArthur ${ }^{4}$, A. McCann ${ }^{16}$, P. Moriarty ${ }^{22}$, R. Mukherjee ${ }^{1}$, R. A. Ong ${ }^{2}$, M. OrR ${ }^{21}$, A. N. Otte ${ }^{3}$, N. Park ${ }^{18}$, J. S. Perkins ${ }^{5}$, A. Pichel ${ }^{23}$, M. Pohl ${ }^{19,24}$, H. Prokoph ${ }^{19}$, J. QuinN ${ }^{8}$, K. Ragan ${ }^{16}$, L. C. Reyes ${ }^{18}$, P. T. Reynolds ${ }^{25}$, E. Roache ${ }^{5}$, H. J. Rose $^{7}$, J. Ruppel ${ }^{24}$, D. B. SAXon ${ }^{17}$, M. Schroedter ${ }^{5}$, G. H. Sembroski ${ }^{11}$, C. Skole ${ }^{19}$, A. W. Smith ${ }^{26}$, D. Staszak ${ }^{16}$, G. Tešić ${ }^{16}$, M. Theiling ${ }^{5}$, S. Thibadeau ${ }^{4}$, K. Tsurusaki ${ }^{14}$, J. Tyler ${ }^{16}$, A. Varlotta ${ }^{11}$, S. VinCent ${ }^{15}$, M. Vivier ${ }^{17}$, S. P. Wakely ${ }^{18}$, J. E. WARD ${ }^{8}$, A. Weinstein ${ }^{2}$, T. WEISGARBER ${ }^{18}$, AND D. A. WILLIAMS ${ }^{3}$

${ }^{1}$ Department of Physics and Astronomy, Barnard College, Columbia University, NY 10027, USA

${ }^{2}$ Department of Physics and Astronomy, University of California, Los Angeles, CA 90095, USA

${ }^{3}$ Santa Cruz Institute for Particle Physics and Department of Physics, University of California, Santa Cruz, CA 95064, USA; apbouvie@ucsc.edu ${ }^{4}$ Department of Physics, Washington University, St. Louis, MO 63130, USA

${ }^{5}$ Fred Lawrence Whipple Observatory, Harvard-Smithsonian Center for Astrophysics, Amado, AZ 85645, USA

${ }^{6}$ Astrophysical Institute, Department of Physics and Astronomy, Ohio University, Athens, OH 45701, USA; boettchm@ohio.edu

${ }^{7}$ School of Physics and Astronomy, University of Leeds, Leeds LS2 9JT, UK

${ }^{8}$ School of Physics, University College Dublin, Belfield, Dublin 4, Ireland

${ }^{9}$ School of Physics, National University of Ireland Galway, University Road, Galway, Ireland

${ }^{10}$ Astronomy Department, Adler Planetarium and Astronomy Museum, Chicago, IL 60605, USA

${ }_{11}$ Department of Physics, Purdue University, West Lafayette, IN 47907, USA

${ }^{12}$ Department of Astronomy and Astrophysics, 525 Davey Lab, Pennsylvania State University, University Park, PA 16802, USA

${ }^{13}$ School of Physics and Astronomy, University of Minnesota, Minneapolis, MN 55455, USA

${ }^{14}$ Department of Physics and Astronomy, University of Iowa, Van Allen Hall, Iowa City, IA 52242, USA; daniel-d-gall@uiowa.edu

${ }^{15}$ Department of Physics and Astronomy, University of Utah, Salt Lake City, UT 84112, USA

${ }^{16}$ Physics Department, McGill University, Montreal, QC H3A 2T8, Canada

${ }^{17}$ Department of Physics and Astronomy and the Bartol Research Institute, University of Delaware, Newark, DE 19716, USA

${ }^{18}$ Enrico Fermi Institute, University of Chicago, Chicago, IL 60637, USA

${ }^{19}$ DESY, Platanenallee 6, 15738 Zeuthen, Germany

${ }^{20}$ Department of Physics and Astronomy, DePauw University, Greencastle, IN 46135-0037, USA

${ }^{21}$ Department of Physics and Astronomy, Iowa State University, Ames, IA 50011, USA

${ }^{22}$ Department of Life and Physical Sciences, Galway-Mayo Institute of Technology, Dublin Road, Galway, Ireland

${ }^{23}$ Instituto de Astronomia y Fisica del Espacio, Casilla de Correo 67-Sucursal 28, (C1428ZAA) Ciudad Autnoma de Buenos Aires, Argentina

${ }^{24}$ Institut für Physik und Astronomie, Universität Potsdam, 14476 Potsdam-Golm, Germany

${ }^{25}$ Department of Applied Physics and Instrumentation, Cork Institute of Technology, Bishopstown, Cork, Ireland

${ }^{26}$ Argonne National Laboratory, 9700 S. Cass Avenue, Argonne, IL 60439, USA

Received 2011 May 25; accepted 2011 July 8; published 2011 August 24

\begin{abstract}
We report on very high energy ( $>100 \mathrm{GeV}$ ) gamma-ray observations of Swift J164449.3+573451, an unusual transient object first detected by the Swift Observatory and later detected by multiple radio, optical, and X-ray observatories. A total exposure of $28 \mathrm{hr}$ was obtained on Swift J164449.3+573451 with the Very Energetic Radiation Imaging Telescope Array System (VERITAS) during 2011 March 28-April 15. We do not detect the source and place a differential upper limit on the emission at $500 \mathrm{GeV}$ during these observations of $1.4 \times 10^{-12} \mathrm{erg} \mathrm{cm}^{-2} \mathrm{~s}^{-1}$ (99\% confidence level). We also present time-resolved upper limits and use a flux limit averaged over the X-ray flaring period to constrain various emission scenarios that can accommodate both the radio-through-X-ray emission detected from the source and the lack of detection by VERITAS.
\end{abstract}

Key words: accretion, accretion disks - galaxies: active - gamma rays: galaxies - radiation mechanisms: non-thermal

\section{INTRODUCTION}

Swift J164449.3+573451 (hereafter Sw J1644+57) was first detected by the Swift Burst Alert Telescope (BAT) on 2011 March 28 at 12:57:45 UT. The Swift spacecraft slewed to the location of the source and began observations with the X-ray Telescope (XRT) and the UV/Optical Telescope (UVOT). These observations located a bright, uncataloged X-ray source but did not identify an optical afterglow typical of gamma-ray bursts (GRBs; Cummings et al. 2011). Less than one hour later, the
BAT triggered a second time on $\mathrm{Sw} \mathrm{J} 1644+57$, which ruled out a GRB origin and gave the first sign of the unusual nature of the source (Kennea et al. 2011a). This prompted multiwavelength follow-up observations at a number of observatories.

These follow-up observations identified an optical source consistent with the position of Sw J1644+57 (Cenko et al. 2011; Leloudas et al. 2011). Measurements obtained with the Gemini Observatory show an infrared (IR) source with a transient component at a location consistent with that of $\mathrm{Sw} \mathrm{J1644+57}$ and provide a redshift of $z=0.3534$ from $\mathrm{H}_{\beta}$ and $\mathrm{O}$ III emission 
lines (Levan et al. 2011a, 2011b). Radio observations with the Enhanced Very Large Array find an unresolved, variable radio source at a position consistent with the optical source detected by Cenko et al. (2011) and Leloudas et al. (2011), suggesting the optical source is most likely the host galaxy (Zauderer et al. 2011a, 2011b; Berger et al. 2011). Hubble Space Telescope observations show a nearly point-like IR source consistent with the location of Sw J1644+57 and, in an optical exposure, a resolved compact galaxy whose nucleus is consistent with the position of the IR point source (Fruchter et al. 2011). Temporal analysis of the Swift light curve, combined with the implied peak luminosity at a distance of $z \sim 0.35$, provides evidence that the observed emission from Sw J1644+57 is likely beamed (Campana et al. 2011; Burrows et al. 2011; Bloom et al. 2011).

Because X-ray and very high energy (VHE; $E>100 \mathrm{GeV}$ ) gamma-ray emission are frequently correlated in other beamed sources, such as blazars (Böttcher 2010), it is reasonable to expect VHE emission from Sw J1644+57, depending on the parameters of the emission region and the surrounding environment. Here, we discuss deep VHE observations of Sw J1644+57 with the Very Energetic Radiation Imaging Telescope Array System (VERITAS) and the implications of our results for some possible emission scenarios for this unusual object.

\section{OBSERVATIONS}

VERITAS is an array of four imaging atmosphericCherenkov telescopes (IACTs) located at the Fred Lawrence Whipple Observatory in southern Arizona at an altitude of $1280 \mathrm{~m}$ above sea level (Holder et al. 2008). Imaging cameras, consisting of 499 photomutiplier tubes located in the focal plane of each telescope, detect Cherenkov light emitted by extensive air showers initiated in the upper atmosphere by gamma rays and cosmic rays. VERITAS has a field of view of 3.5 and is sensitive in the range of $100 \mathrm{GeV}-30 \mathrm{TeV}$. The telescopes typically operate in "wobble" mode, where the location of the target is offset from the center of the field of view by 0.5 , allowing for simultaneous background measurements (Fomin et al. 1994). The offset direction alternates between north, south, east, and west for each data segment (typically lasting 20 minutes) to reduce systematic errors in the background estimation.

On 2011 March 29 at 10:27 UT, approximately $22.5 \mathrm{hr}$ after the first BAT trigger, VERITAS started observing Sw J1644+57. Subsequent daily observations with an average exposure of $\sim 2 \mathrm{hr} /$ night were taken when weather conditions were favorable, continuing through 2011 April 15, after which observations were not possible because of the near-full Moon (exceeding $\sim 97 \%$ illumination). Zenith angles for our observations ranged from $25^{\circ}$ to $40^{\circ}$. Due to temporary hardware issues, approximately $15 \%$ of the data were taken with an array of three telescopes. In total, VERITAS accumulated $\sim 28 \mathrm{hr}$ of exposure on this source, of which $\sim 3.5 \mathrm{hr}$ were taken within one day of the particularly intense flaring events observed in X-rays during 2011 March 28-31 (Kennea et al. 2011b).

For this analysis, about $90 \%$ of the data $(\sim 25 \mathrm{hr})$ pass the quality selection criteria, with selection based primarily on weather conditions and trigger-rate stability. The selected data are processed through the standard VERITAS analysis package (Cogan 2007). Our cosmic-ray rejection procedure is based on applying selection criteria on standard image parameters (Hillas 1985): the size of the telescope images, the mean scaled width and mean scaled length parameters (Krawczynski et al. 2006), the height of maximum Cherenkov emission and the angular
Table 1

Selection Criteria Used for VERITAS Analysis

\begin{tabular}{lc}
\hline \hline Parameter & Selection Criteria \\
\hline Image size & $>400$ digital counts $(\sim 75$ photoelectrons $)$ \\
Mean scaled width & $0.05<\mathrm{MSW}<1.15$ \\
Mean scaled length & $0.05<\mathrm{MSL}<1.3$ \\
Height of shower maximum & $>7 \mathrm{~km}$ \\
$\theta$ & $<0.1$ \\
\hline
\end{tabular}

Note. For an explanation of these parameters see Section 2.

distance from the putative source position to the reconstructed arrival direction of the shower $(\theta)$. The standard selection criteria (see Table 1) were optimized using Monte Carlo simulations and real data from the Crab Nebula and the blazar PG $1553+113$.

The remaining background is estimated using the "reflectedregion" method described in Berge et al. (2007). The radii of the circular on- and off-source regions are 0.1 . Statistical significances are computed using a modified version of Equation 17 from Li \& Ma (1983) to allow for varying number of off-source regions due to the bright ( $V=4.849)$ nearby star HR 6237 (Aharonian et al. 2004).

\section{RESULTS}

Significant VHE gamma-ray emission is not detected from the direction of $\mathrm{Sw} \mathrm{J1644+57}$ in the entire data set nor in subsets of the data (see Table 2). In order to look specifically for VHE emission contemporaneous with the intense X-ray flaring, the first subset consists of data that were taken within one day before or after periods where the XRT count rate exceeded $20 \mathrm{~s}^{-1}$. This subset is denoted the "flaring" period and comprises the first three nights of observations. However, it is worth noting that VERITAS exposures during this "flaring" period fell between X-ray flares observed by Swift. Therefore, VERITAS observations were simultaneous with relatively low X-ray flux states during that period, characterized by an X-ray flux of $v F_{v} \sim 10^{-10} \mathrm{erg} \mathrm{cm}^{-2} \mathrm{~s}^{-1}$, about two orders of magnitude lower than the major flares. The second subset, denoted the "low" period, comprises the remainder of the data.

Following the lack of signal in the data, we derive $99 \%$ confidence level upper limits over various time intervals (see Table 2).

1. Total: 2011 March 29-April 15.

2. Flaring: 2011 March 29-31.

3. Low: 2011 April 1-15.

4. Daily (by UT date, when observations available).

The procedure described by Rolke et al. (2005) is chosen for the upper-limit computation with the assumption of a Gaussiandistributed background. The total, flaring and low-flux upper limits on $E^{*} F(E)$ at $99 \%$ c.l. are $1.4 \times 10^{-12}, 3.1 \times 10^{-12}$, and $1.5 \times 10^{-12} \mathrm{erg} \mathrm{cm}^{-2} \mathrm{~s}^{-1}$, respectively, where $F(E)$ is the energy flux. The limits are calculated at $500 \mathrm{GeV}$ assuming any emission follows a power-law spectrum with a photon index of -3.0 . The decorrelation energy $(500 \mathrm{GeV})$ is used to reduce the sensitivity of the limits to the choice of photon index; this energy is higher than the energy threshold of the observations $(\sim 290 \mathrm{GeV})$. The flaring and low-state upper limits along with the daily upper limits are presented in Figure 1, superimposed on the Swift XRT light curve (Evans et al. 2007) for comparison. 


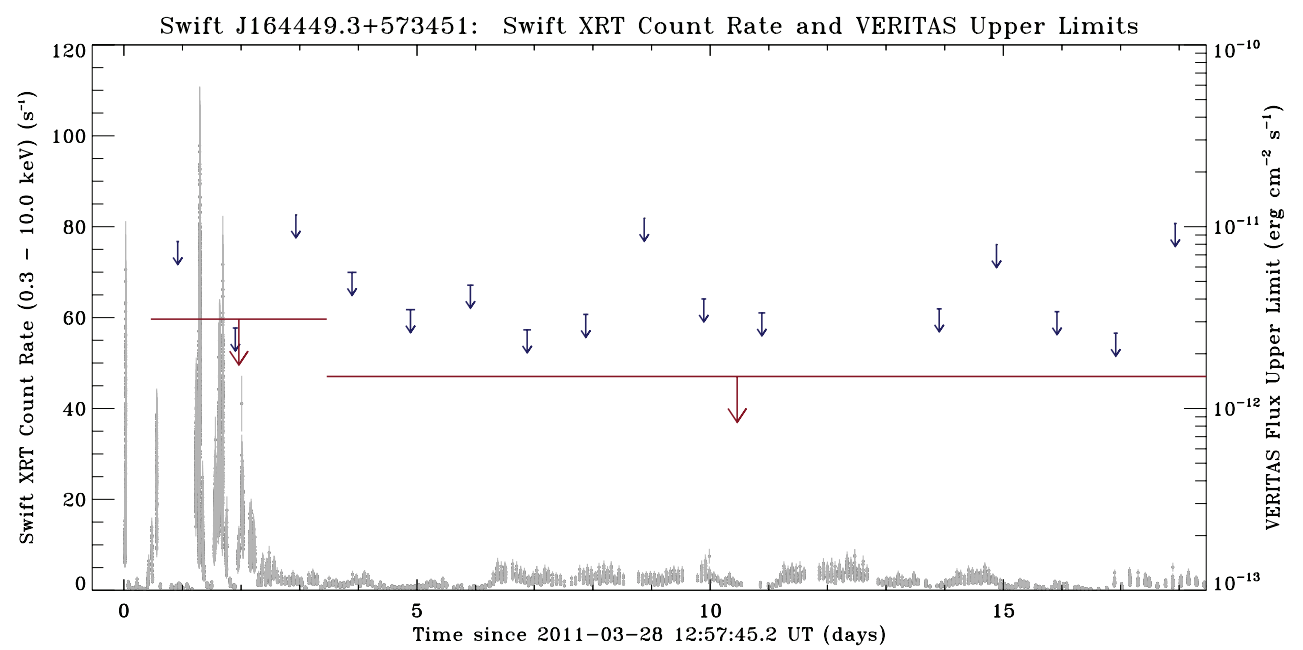

Figure 1. Flaring, low-state and daily upper limits are shown superimposed on the Swift XRT light curve (Evans et al. 2007). The extent of the daily upper limit horizontal bars represents the approximate time interval during which the VERITAS observations were taken.

Table 2

Data Analysis Results

\begin{tabular}{lccc}
\hline \hline & Total & Flaring & Low \\
\hline Date range & 2011 Mar 29-Apr 15 & 2011 Mar 29-31 & 2011 Apr 1-15 \\
ON (source) counts & 579 & 59 & 520 \\
OFF (background) counts & 5639 & 604 & 5035 \\
Average $\alpha$ & 0.1 & 0.1 & 0.1 \\
Significance & $0.6 \sigma$ & $-0.3 \sigma$ & $0.8 \sigma$ \\
Excess counts & 15.1 & -1.4 & 16.5 \\
Flux upper limit (99\% c.l.) $\left[E * F(E) ; \mathrm{erg} \mathrm{cm}^{-2} \mathrm{~s}^{-1}\right]$ & $1.4 \times 10^{-12}$ & $3.1 \times 10^{-12}$ & $1.5 \times 10^{-12}$ \\
\hline
\end{tabular}

Notes. Flux upper limits calculated assuming a photon power-law index of -3.0 and taken at the decorrelation energy of $500 \mathrm{GeV}$. The ratio of on-source to off-source exposure is denoted as $\alpha$.

\section{DISCUSSION}

In this section, we provide some generic parameter constraints that can be derived from the observed X-ray properties of Sw J1644+57, along with the non-detection by Fermi/LAT (Omodei et al. 2011) and VERITAS. The X-ray flux varied on timescales of $t_{\mathrm{var}}=100 \mathrm{~s}$ (Burrows et al. 2011), with a peak energy flux of $F_{X} \sim 10^{-8} \mathrm{erg} \mathrm{cm}^{-2} \mathrm{~s}^{-1}$, corresponding to a peak luminosity of $L_{\mathrm{pk}} \sim 4.3 \times 10^{48} \mathrm{erg} \mathrm{s}^{-1}$ if the emission were isotropic. In order to illustrate the dependence of the following estimates on the variability timescale, we parameterize $t_{\mathrm{var}} \equiv 100 t_{\mathrm{var}, 2} \mathrm{~s}$. The Eddington limit implies a central engine mass of $M>3.4 \times 10^{10} M_{\odot}$, assuming unbeamed emission. Assuming that the emission-region size is not smaller than the Schwarzschild radius of the central engine, the observed variability implies $M<10^{7} M_{\odot}$. The two mass estimates can be reconciled by allowing for anisotropic and/or beamed emission, plausibly involving relativistic motion. Relativistic motion will result in Doppler boosting of the luminosity by a factor $D^{4}$, along with variability time contraction by a factor $D^{-1}$, where $D=\left(\Gamma\left[1-\beta_{\Gamma} \cos \theta\right]\right)^{-1}$ is the Doppler factor, $\Gamma=\left(1-\beta_{\Gamma}^{2}\right)^{-1 / 2}$ is the bulk Lorentz factor of the emission region, $\beta_{\Gamma} c$ is its velocity, and $\theta$ is the angle between the direction of motion and the line of sight. Reconciling the mass estimates above requires Doppler boosting by at least a factor $D>5.4$.

Bloom et al. (2011) and Burrows et al. (2011) have argued that this event arises from the activation of a beamed jet and have hypothesized that this may be the result of tidal disruption of a star by a $\sim 10^{6}-10^{7} M_{\odot}$ black hole. Both synchrotrondominated (Burrows et al. 2011) and Compton-dominated
(Bloom et al. 2011) origins have been proposed for the X-ray emission. Burrows et al. (2011) propose a Poynting-fluxdominated scenario, in which the $\mathrm{X}$-ray emission is produced by synchrotron emission from relativistic electrons. Bloom et al. (2011) interpret the lack of variability of the radio-IR emission as evidence that the radio-IR emission is produced in a more extended region than the X-rays. They suggest inverse Compton scattering of external radiation as the mechanism producing the high-energy radiation.

In the following discussion, we present some general considerations to constrain the parameters of the X-ray emission region, including constraints placed by the VHE upper limits. We consider both synchrotron and inverse Compton as possible emission mechanisms.

\subsection{Synchrotron Origin}

We first consider a scenario in which the X-ray emission is synchrotron emission by relativistic electrons in a tangled magnetic field $B$. The electron Lorentz factor at which the nonthermal electron distribution has its peak radiative output is $\gamma_{p}$, and the electron density at that energy is $n_{p} \equiv n_{e}\left(\gamma_{p}\right)$. The observed spectral variability suggests that the peak frequency might vary substantially during the various outbursts. For the following estimates, we scale the peak frequency as $v_{\mathrm{pk}} \equiv 10^{19} v_{p, 19} \mathrm{~Hz}$ and we base our estimates on the typical $\mathrm{X}$-ray flux observed during VERITAS observations in the flaring state $v F_{v}^{\mathrm{sy}} \equiv 10^{-10} f_{-10} \mathrm{erg} \mathrm{cm}^{-2} \mathrm{~s}^{-1}$, corresponding to $v L_{v}=4.3 \times 10^{46} \mathrm{erg} \mathrm{s}^{-1}$. We further assume that the variability timescale provides an estimate of the emission-region size, $R_{B}=c t_{\mathrm{var}} D /(1+z)$. The observables can then be related to 
the emission-region parameters through (Rybicki \& Lightman 1979)

$$
\begin{aligned}
v_{\mathrm{pk}} & =4.2 \times 10^{6} \gamma_{p}^{2}\left(\frac{B}{1 \mathrm{G}}\right) \frac{D}{1+z} \mathrm{~Hz}, \\
v L_{v} & =\frac{2}{9} c \sigma_{T} B^{2} \gamma_{p}^{2} n_{p}\left(\frac{c t_{\mathrm{var}}}{1+z}\right)^{3} D^{7} .
\end{aligned}
$$

A further constraint is derived from the condition that the synchrotron cooling timescale of electrons of energy $\gamma_{p}$ should be of the order of the observed variability timescale. This corresponds to the assumption that the entire energy transferred to radiation throughout the duration of the flare is contained in the particle population at the onset of the flare. We define such a scenario as a particle-dominated scenario. Burrows et al. (2011) have shown that an alternative, Poynting-flux-dominated scenario with synchrotron cooling timescales of the order of $t_{\text {sy }} \lesssim 0.1 \mathrm{~s}$ can explain the spectral energy distribution (SED)

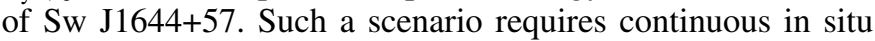
re-acceleration of electrons to maintain a low-energy cutoff in the electron distribution, which is needed in order to reproduce the observed hard optical-X-ray spectral slope.

Assuming that the electron cooling timescale and the lightcrossing timescale across the source are of the same order, we estimate

$$
t_{\mathrm{var}} \sim t_{\mathrm{sy}} \frac{1+z}{D}=\frac{6 \pi m_{e} c^{2}}{c \sigma_{T} B^{2} \gamma_{p}} \frac{1+z}{D}
$$

Parameterizing the Doppler factor in terms of $D_{1} \equiv D / 10$, we solve Equations (1)-(3) to find

$$
\begin{gathered}
B=1.5 D_{1}^{-1 / 3} v_{p, 19}^{-1 / 3} t_{\mathrm{var}, 2}^{-2 / 3} \mathrm{G}, \\
\gamma_{p}=4.4 \times 10^{5} D_{1}^{-1 / 3} t_{\mathrm{var}, 2}^{1 / 3} v_{p, 19}^{2 / 3}, \\
n_{p}=6.7 \times 10^{4} D_{1}^{-17 / 3} v_{p, 19}^{-2 / 3} t_{\mathrm{var}, 2}^{-7 / 3} f_{-10} \mathrm{~cm}^{-3} .
\end{gathered}
$$

These parameters correspond to a Thomson depth from electrons near the peak, $\tau_{T}$, of

$$
\tau_{T}=n_{e} \sigma_{T} R_{B}=10^{-6} D_{1}^{-14 / 3} v_{p, 19}^{-2 / 3} t_{\mathrm{var}, 2}^{-4 / 3} f_{-10}
$$

The expected synchrotron radiation energy density in the comoving frame, $u_{\mathrm{sy}}^{\prime}$, is

$$
u_{\mathrm{sy}}^{\prime} \sim \frac{16}{9} \tau_{T} \gamma_{p}^{2} u_{B}^{\prime}
$$

where $u_{B}^{\prime}=B^{2} /(8 \pi)$ is the comoving magnetic-field energy density. Electrons with energy corresponding to $\gamma_{p}$ will encounter synchrotron photons at frequency $\nu_{\mathrm{pk}}$ (observer's frame) in the extreme Klein-Nishina limit, $\gamma_{p} \epsilon_{\mathrm{pk}}^{\prime} \sim 3.5 \times$ $10^{3} D_{1}^{-4 / 3} t_{\mathrm{var}, 2}^{1 / 3} v_{p, 19}^{5 / 3}$ where $\epsilon_{\mathrm{pk}}^{\prime}=h v_{\mathrm{pk}} /\left(D m_{e} c^{2}\right)$. Therefore, the inverse Compton output from electrons at $\gamma_{p}$ is strongly suppressed by a factor $f_{\mathrm{KN}}=\frac{3}{8} \frac{\ln \left(2 \epsilon^{\prime} \gamma\right)+1 / 2}{\epsilon^{\prime} \gamma} \sim 7.6 \times$ $10^{-4} D_{1}^{4 / 3} t_{\mathrm{var}, 2}^{-1 / 3} v_{p, 19}^{-5 / 3}$. Hence, the ratio of synchrotron selfCompton (SSC) to synchrotron luminosities is expected to be

$$
\left(\frac{L_{\mathrm{SSC}}}{L_{\mathrm{sy}}}\right)=\frac{u_{\mathrm{sy}}^{\prime}}{u_{B}^{\prime}} \times f_{\mathrm{KN}} \sim 2.6 \times 10^{2} D_{1}^{-4} t_{\mathrm{var}, 2}^{-1} v_{p, 19}^{-1} f_{-10},
$$

which leads to an estimated intrinsic VHE flux of

$$
\begin{aligned}
v F_{v}^{\mathrm{SSC}, \text { int }}= & \left(\frac{L_{\mathrm{SSC}}}{L_{\mathrm{sy}}}\right) v F_{v}^{\mathrm{sy}} \sim 2.6 \times 10^{-8} \\
& \times D_{1}^{-4} t_{\mathrm{var}, 2}^{-1} v_{p, 19}^{-1} f_{-10}^{2} \mathrm{erg} \mathrm{cm}^{-2} \mathrm{~s}^{-1},
\end{aligned}
$$

with an emission peak around $D \gamma_{p} m_{e} c^{2} /(1+z) \sim 1.7 D_{1}^{2 / 3}$ $t_{\mathrm{var}, 2}^{1 / 3} v_{p, 19}^{2 / 3} \mathrm{TeV}$. We point out that if $L_{\mathrm{SSC}} / L_{\mathrm{sy}}>1$, the effective electron cooling timescale will actually be shorter than the synchrotron cooling timescale by a factor $\sim L_{\mathrm{sy}} / L_{\mathrm{SSC}}$, in which case our initial assumption $t_{\mathrm{var}} \sim t_{\mathrm{sy}} \frac{1+z}{D}$ breaks down.

At VHE photon energies, the effect of $\gamma \gamma$ absorption internal to the emission region may become substantial (Burrows et al. 2011). Photons of comoving photon energy $\epsilon^{\prime}{ }_{\gamma} \equiv h \nu^{\prime}{ }_{\gamma} /\left(m_{e} c^{2}\right) \equiv 10^{5} \epsilon_{6} D_{1}^{-1}$ are most efficiently absorbed by target photons of energy $\epsilon^{\prime}{ }_{T} \sim 2 / \epsilon^{\prime}{ }_{\gamma}$, corresponding to an observed target photon frequency of $\nu_{T} \sim 10^{16} D_{1}^{2} \epsilon_{6}^{-1} \mathrm{~Hz}$, i.e., UV photons. We note that the UV flux appears to be very strongly absorbed by gas and dust local to the host galaxy, and the intrinsic UV flux may well be several orders of magnitude higher than the UVOT upper limits of $\lesssim 10^{-14} \mathrm{erg} \mathrm{cm}^{-2} \mathrm{~s}^{-1}$. Here we parameterize the intrinsic flux of photons at those frequencies as $\nu F_{v}\left(\epsilon_{T}\right) \equiv 10^{-11} f_{\mathrm{UV},-11} \mathrm{erg} \mathrm{cm}^{-2} \mathrm{~s}^{-1}$ since the $\mathrm{X}$-ray to optical flux extrapolations from the SED, as well as other evidence about the extinction, show that $f_{\mathrm{UV}}$ is probably around $0.5 \times 10^{-11} \mathrm{erg} \mathrm{cm}^{-2} \mathrm{~s}^{-1}$ (Burrows et al. 2011). Based on a $\delta$-function approximation to the $\gamma \gamma$ absorption cross section, the optical depth for $\gamma \gamma$ absorption is estimated as

$$
\begin{aligned}
\tau_{\gamma \gamma}^{\mathrm{int}} & \sim \frac{4}{3} \frac{\sigma_{T} d_{L}^{2} \nu F_{v}\left(\epsilon_{T}\right)(1+z)}{\epsilon_{T}^{\prime} D^{5} m_{e} c^{2} c^{2} t_{\mathrm{var}}} \\
& \sim 5.5 \times 10^{3} f_{\mathrm{Uv},-11} \epsilon_{6} D_{1}^{-6} t_{\mathrm{var}, 2}^{-1} .
\end{aligned}
$$

For internal absorption, the suppression of the flux is given by $F_{\text {abs,internal }}=F_{\text {internal }}\left(1-e^{-\tau_{\gamma \gamma}^{\text {int }}}\right) / \tau_{\gamma \gamma}^{\text {int }} \approx F_{\text {int }} / \tau_{\gamma \gamma}^{\text {int }}$ for $\tau_{\gamma \gamma}^{\text {int }} \gg 1$. Consequently, after correction for extinction by the extragalactic background light (EBL), which amounts to a factor of $e^{-\tau_{\gamma \gamma}^{\mathrm{EBL}}} \sim$ $1 / 13$ at $500 \mathrm{GeV}$, using the Finke et al. (2010) EBL model, the particle-dominated synchrotron scenario predicts a VHE $\gamma$-ray flux of $\nu F_{v}^{\mathrm{SSC} \text {,int }} e^{-\tau_{\gamma \gamma}^{\mathrm{EBL}}} / \tau_{\gamma \gamma}^{\mathrm{int}} \sim 3.6 \times 10^{-13} \mathrm{erg} \mathrm{cm}^{-2} \mathrm{~s}^{-1}$, which is slightly below the VERITAS upper limits.

However, such an emission model would require the following equipartition ratio between the comoving energy densities in the magnetic field, $u_{B}^{\prime}$, and the relativistic electrons, $u_{e}^{\prime}$, based on the parameters of Equations (4)-(6):

$$
\epsilon_{B} \equiv u_{B}^{\prime} / u_{e}^{\prime}=3.7 \times 10^{-6} D_{1}^{16 / 3} v_{p, 19}^{-2 / 3} t_{\mathrm{var}, 2}^{2 / 3} f_{-10}^{-1} .
$$

Therefore, the particle-dominated synchrotron scenario, though possible, is disfavored as it requires an unusually large Doppler factor ( $\gtrsim 100)$ to allow for equipartition to occur in the jet.

\subsection{External Inverse Compton Origin}

The X-rays may also be produced by inverse Compton scattering of low-energy radiation. We scale the peak frequency of the soft target photons for Compton scattering as $v_{s} \equiv$ $10^{13} v_{s, 13} \mathrm{~Hz}$. Assuming that the external radiation field is approximately isotropic in the rest frame of the host galaxy, the observed Compton peak frequency is $v_{\mathrm{pk}} \sim v_{s} \gamma_{p}^{2} D^{2} /(1+z) \sim$ $10^{19} v_{p, 19} \mathrm{~Hz}$, yielding

$$
\gamma_{p} \sim 10^{2} v_{s, 13}^{-1 / 2} D_{1}^{-1} v_{p, 19}^{1 / 2}
$$


Setting the observed variability timescale equal to the Compton cooling timescale (modulo $D /(1+z)$ ) yields an estimate of the energy density of the external radiation field in the comoving frame,

$$
u_{s}^{\prime} \sim 415 v_{s, 13}^{1 / 2} v_{p, 19}^{-1 / 2} t_{\mathrm{var}, 2}^{-1} \mathrm{erg} \mathrm{cm}^{-3},
$$

which is related to the energy density in the rest frame of the host galaxy through $u_{s} \approx u_{s}^{\prime} / \Gamma^{2} \sim u_{s}^{\prime} / D^{2}$. Assuming that this emission originates within a few hundred Schwarzschild radii of the central black hole (i.e., $R_{\mathrm{ext}}=10^{15} R_{15} \mathrm{~cm}$ ), the above radiation energy density results in a luminosity of $L_{s} \sim 4 \pi R_{\mathrm{ext}}^{2} c u_{s} \sim 1.6 \times 10^{42} R_{15}^{2} D_{1}^{-2} t_{\mathrm{var}, 2}^{-1} v_{s, 13}^{1 / 2} v_{p, 19}^{-1 / 2} \mathrm{erg} \mathrm{s}^{-1}$, which corresponds to a flux of

$$
v F_{v}^{\mathrm{s}} \sim 3.8 \times 10^{-15} R_{15}^{2} D_{1}^{-2} t_{\mathrm{var}, 2}^{-1} v_{s, 13}^{1 / 2} v_{p, 19}^{-1 / 2} \mathrm{erg} \mathrm{cm}^{-2} \mathrm{~s}^{-1},
$$

which would be of the order of the observed flux of the IR peak for $R_{15} \sim$ a few. The observed X-ray peak luminosity can be used analogous to Equation (2) to infer the density of electrons around $\gamma_{p}$ :

$$
n_{p} \sim 8.5 \times 10^{6} f_{-10} v_{s, 13}^{1 / 2} D_{1}^{-7} v_{p, 19}^{-1 / 2} t_{\mathrm{var}, 2}^{-2} \mathrm{~cm}^{-3},
$$

which yields a Thomson depth of

$$
\tau_{T} \sim 1.3 \times 10^{-4} f_{-4} v_{s, 13}^{1 / 2} D_{1}^{-6} v_{p, 19}^{-1 / 2} t_{\mathrm{var}, 2}^{-1}
$$

Analogous to Equations (8) and (9), we can now compute the expected importance of higher-order Compton scatterings through the ratio of luminosities in second-order to first-order Compton luminosities:

$$
\left(\frac{L_{C 2}}{L_{C 1}}\right)_{\text {Thomson }} \sim 2.2 f_{-10} D_{1}^{-8} v_{s, 13}^{-1 / 2} v_{p, 19}^{1 / 2} t_{\mathrm{var}, 2}^{-1},
$$

and this emission would peak at

$$
v_{C 2} \sim 10^{21} D_{1}^{-2} v_{s, 13}^{-1} v_{p, 19}^{2} \mathrm{~Hz}
$$

which corresponds to $E_{C 2} \sim 4 D_{1}^{-2} v_{s, 13}^{-1} v_{p, 19}^{2} \mathrm{MeV}$ and is substantially below the Fermi/LAT regime of $E>100 \mathrm{MeV}$. We therefore conclude that higher-order Compton scattering is not expected to lead to a detectable signal in the Fermi/LAT or VERITAS regimes.

Bloom et al. (2011) suggested that the target field for Compton scattering might be UV-soft X-ray emission from an accretion disk formed during the tidal disruption event. This would correspond to $v_{s, 13} \sim 10^{4}$. Consequently, the X-rays could be produced through the bulk Compton process by cold (in the comoving frame) electrons. We would then infer an external radiation energy density of $u_{s} \sim 420 D_{1}^{-2} t_{\mathrm{var}, 2}^{-1} v_{p, 19}^{-1 / 2} \mathrm{erg} \mathrm{cm}^{-3}$, corresponding to an observed flux of $v F_{v}^{s} \sim 3.8 \times$ $10^{-13} R_{15}^{2} D_{1}^{-2} t_{\text {var, } 2}^{-1} v_{p, 19}^{-1 / 2}$ erg $\mathrm{cm}^{-2} \mathrm{~s}^{-1}$. Considering the substantial UV extinction toward the emission region, this flux still appears consistent with the UVOT upper limits.

We conclude that among the scenarios discussed here, inverse Compton scattering of either the observed radio-IR radiation by relativistic electrons of $\gamma_{p} \sim 10^{2}$ or of a putative accretionrelated UV radiation field scattered by cold electrons in a relativistically moving emission region with $D \sim 10$ are plausible mechanisms for the production of the observed rapidly-varying $\mathrm{X}$-ray emission and are compatible with the Fermi-LAT and VERITAS upper limits. As discussed by Burrows et al. (2011), the observed SED and variability are also consistent with a synchrotron-dominated X-ray emission scenario, if the jet has a strong magnetic field (Poynting-flux-dominated) and has ongoing in situ acceleration of electrons. However, if the energy content of the emission region is dominated by relativistic particles, either far sub-equipartition magnetic fields or an uncomfortably large Doppler factor are required.

This research is supported by grants from the U.S. Department of Energy, the U.S. National Science Foundation and the Smithsonian Institution, by NSERC in Canada, by Science Foundation Ireland (SFI 10/RFP/AST2748), and by STFC in the UK. We acknowledge the excellent work of the technical support staff at the Fred Lawrence Whipple Observatory and the collaborating institutions in the construction and operation of the instrument. This work made use of data supplied by the UK Swift Science Data Centre at the University of Leicester.

\section{REFERENCES}

Aharonian, F., Akhperjanian, A., Beilicke, M., et al. 2004, A\&A, 421, 529 Berge, D., Funk, S., \& Hinton, J. 2007, A\&A, 466, 1219

Berger, E., Levan, A., Tanvir, N. R., et al. 2011, GCN Circ., 11854

Bloom, J. S., Giannios, D., Metzger, B. D., et al. 2011, submitted (arXiv: 1104.3257)

Böttcher, M. 2010, in Proc. Fermi Meets Jansky, ed. T. Savolainan, E. Ros, R. W. Porcas, \& J. A. Zensus (Bonn: MPIfR), 41

Burrows, D. N., Kennea, J. A., Ghisellini, G., et al. 2011, submitted (arXiv: 1104.4787)

Campana, S., Covino, S., Tagliaferri, G., et al. 2011, GCN Circ., 11843

Cenko, S. B., Nugent, P. E., Fox, D. B., et al. 2011, GCN Circ., 11827

Cogan, P. (for the VERITAS Collaboration) 2007, Proc. 30th ICRC (Mérida), 3,1385

Cummings, J. R., et al. 2011, GCN Circ., 11823

Evans, P. A., Beardmore, A. P., Page, K. L., et al. 2007, A\&A, 469, 379

Finke, J. D., Razzaque, S., \& Dermer, C. D. 2010, ApJ, 712, 238

Fomin, V. P., Stepanian, A. A., Lamb, R. C., et al. 1994, Astropart. Phys., 2, 137

Fruchter, A. S., Misra, K., Graham , J., et al. 2011, GCN Circ., 11881

Hillas, A. M. 1985, Proc. 19th ICRC (La Jolla), 3, 445

Holder, J., et al. 2008, in AIP Conf. Proc. 1085, High Energy Gamma-Ray Astronomy, ed. F. A. Aharonian, W. Hofmann, \& F. Rieger (Melville, NY AIP), 657

Kennea, J. A., Romano, P., Krimm, H. A., et al. 2011a, ATel, 3242

Kennea, J. A., Romano, P., Krimm, H. A., et al. 2011b, ATel, 3250

Krawczynski, H., Carter-Lewis, D. A., Duke, C., et al. 2006, Astropart. Phys., 25,380

Leloudas, G., Malesani , D., Tanvir, N. R., et al. 2011, GCN Circ., 11830

Levan, A. J., Perley, D., Tanvir, N. R., et al. 2011a, GCN Circ., 11853

Levan, A. J., Tanvir, N. R., Cenko, S. B., et al. 2011b, Science, 333, 199

Li, T., \& Ma, Y. 1983, ApJ, 272, 317

Omodei, N., Troja, E., Corbet, R., et al. 2011, GCN Circ., 11862

Rolke, W. A., López, A. M., \& Conrad, J. 2005, Nucl. Instrum. Methods Phys. Res. A, 551, 493

Rybicki, G. B., \& Lightman, A. P. 1979, Radiative Processes in Astrophysics (New York: Wiley)

Zauderer, A., Berger, E., Frail, D. A., et al. 2011a, GCN Circ., 11836

Zauderer, A., Berger, E., Frail, D. A., et al. 2011b, GCN Circ., 11848 